\title{
A Pilot Correlation Study of Gut Microbiome between Children with Henoch-Schönlein Purpura and their Mothers
}

\section{Changying Zhao}

qilu children's hospital of shandong university

\section{Ying Wang}

qilu children's hospital of shandong university

Junjie Yang

Qilu Normal University

Jiaming Zhang

Shandong University

Xuemei Liu

qilu children's hospital of shandong university

Hongxia Zhang

qilu children's hosipital of shandong university

Xingcui Wang

qilu children's hospital of shandong university

\section{Nan Shen}

jinan children's hospital

\section{Bin Chen}

Beihang University

Yi Liu

jinan children's hospital

Lei Zhang ( $\nabla$ microbiome@foxmail.com)

Beihang

Zhongtao Gai

Shandong University

\section{Research article}

Keywords: Henoch-Schönleinpurpura, Gut microbiome, Bacterial biomarker, Vertical microbiome transmission, Microbiota dysbiosis

Posted Date: May 6th, 2020 
DOI: https://doi.org/10.21203/rs.3.rs-26036/v1

License: (c) (1) This work is licensed under a Creative Commons Attribution 4.0 International License. Read Full License 


\section{Abstract}

Background: Vertical microbiome transmission from mothers plays an important role inchildren with the establishment and development of gutmicrobiome.Our previous study has found that gut microbiota dysbiosis is associated with Henoch-Schönleinpurpura(HSP) in children,while it is unclear whether there is a correlation between the gut microbiome of the HSP children and their mothers.

Methods: In this study, 50HSP child-mother pairs and 61 matched healthy child-mother pairs were enrolled and examined.Stool samples were collected for DNA extraction and 16S rRNA genesequencing, followed by analyses of the gut microbiota composition.

Results: Significant differences were observed in the gut microbiome composition between HSP children and healthy children. Several unique biomarkers, such as Enterococcus, Fusobacterium, Veillonellaand Streptococcus were identified. A significant increase of the relative abundance of Prevotella and Megamonas were observed in HSP's mothers (HSP-M) compared with mothers of the healthy children ( $\mathrm{H}-$ M). HSP children's gut microbiome is closely associated with that of their mothers, and the taxon of AcidaminococcusandRoseburiawere onlyfound in the gut microbiome of HSP children and their mothers (HSP-M+C).

Conclusions: Our preliminary study revealed that the gut microbiome of HSP children are closely associated with that of their mothers, although children with HSP still possess unique bacterial biomarkers. Dysbiosis of the maternal gut microbiota may play a critical role in increasing the risk of HSP in children, which deserve further longitudinalinvestigations to uncover its mechanisms.

\section{Background}

Henoch-Schönlein purpura (HSP) is the most common type of vasculitis in children. It is often a selflimited and benign disease, and the highest incidence is among children aged 4 to 6 years(1). Systemic involvement is very frequent, and complications may occur, such as renal involvement (2). HSP is clinically characterized by abdominal pain, predominance of IgA deposition on a biopsy specimen, arthritis or arthralgia, or renal involvement indicated by hematuria/proteinuria(3). In most cases the patient presents with symptoms characteristic of the disease, and the diagnosis is readily made. Immune, genetic and environmental factors all play a role in the pathogenesis of HSP(4).

The gut microbiome is widely considered to be closely linked to human health, which are thought to be have marked effects on the immune system in health and disease(5). Many immune diseases are reported to be associated with gut microbiome. such as atopic eczema and Kawasaki disease $(6,7)$. In our previous study, we found gut microbiota dysbiosis in children with HSP, and the gut microbiome was associated with the clinical indices(8). Recent studies suggested that the maternal microbiome reservoir play a crucial role in infant microbiome's development. Maternal skin and vaginal strains colonize only transiently, while maternal gut strains proved more persistent in the infant gut and ecologically better adapted than those acquired from other sources(9). Bacterial community acquisition in the infant gut 
impacts immune education and disease susceptibility. In most cases, the child inherits the mother's dominant bacteria strain(10), or selectively inherit bacteria strains according to their function (11). It becomes important to investigate the microbiome association between HSP children and their mothers to evaluate whether vertical microbiome transmission occurs. In the current study, we assessed the gut microbiota by high-throughput sequencing of the 16S ribosomal RNA (16S rRNA) gene in fecal samples from $50 \mathrm{HSP}$ child-mother pairs and 61 matched healthy mother-child pairs and compared overall microbiome composition, and variations of specific bacterial taxa.

\section{Methods}

\section{Study population}

This study was approved by the Institutional Review Boards of Qilu Children's Hospital of Shandong University (QCH IRB\# 16-024), and sample collection began in July 2016. Written informed consent and questionnaire data sheets were obtained from all participants (mothers) who visited the Institute of Child Health Care and agreed to donate stool samples, in compliance with national legislation and the Code of Ethical Principles for Medical Research Involving Human Subjects of the World Medical Association (Declaration of Helsinki). We collected stool samples from 50 HSP mother-child pairs and 61 matched healthy mother-child pairs for the current gut microbiome study. HSP children were enrolled per the following criteria: no infectious disease, malnutrition, immune system defects, congenital genetic metabolic disorders, or treatment with immunoglobulins or immunomodulating agents (12). All HSP children were sampled before administration, without antibiotic or probiotic treatment. Age-matched healthy children were recruited as controls, and physical examination was preformed to ensures that they have no genetic history and other inflammation. All the mothers had passed the physical examination without HSP, not pregnant, HIV negative, without clinically evident inflammatory conditions, didn't use any oral or intravaginal antibiotic within five weeks at the start of the study. All subjects were not malnourished or obese, with normal body mass index.

\section{Sample collection, DNA extraction, and 16S rRNA gene amplicon sequencing}

Stool samples from enrolled patients were collected with sterilized 2-ml tubes containing pure ethanol, and immediately frozen in the freezer at $-80^{\circ} \mathrm{C}$. Genomic DNA was extracted using the $2 \times \mathrm{CTAB}$ (cetyltrimethylammonium ammonium bromide), phenol chloroform mixture (phenol: chloroform: isoamyl alcohol = 25: 24: 1). In the next steps of DNA isolation, we used spin column from SanPrep Column DNA Gel Extraction Kit (Sangon Biotech, China) (based on a silica gel membrane) to purify and recover the DNA rapidly. DNA concentration was measured by NanoDrop ND-2000 (Thermo Fisher Scientific).

To analyze the microbial community within the samples, the $\mathrm{V} 1-\mathrm{V} 2$ hyper-variable region of the bacterial 16S rRNA gene was performed $(8,13)$. Two universal bacterial $16 \mathrm{~S}$ rRNA gene amplicon PCR primers (PAGE purified) were used: forward primer-27F (5'-AGAGTTTGATCMTGGCTCAG3') and reverse primer- 
355R (5'-GCTGCCTCCCGTAGGAGT3'). Amplicons were purified with QIAquick PCR Purification Kit (Qiagen) PCR purification procedure. All amplicons were quantified and pooled to equalize concentrations for sequencing using HiSeq 2500 (Illumina).

\section{S rRNA gene amplicon sequencing analysis}

The 16S rRNA gene sequence paired-end data set was joined and quality filtered using FLASH method (14). All sequences analysis was provided in the Quantitative Insights Into Microbial Ecology (QIIME, version 1.9.1) software suite (15). Chimeric sequences were removed using usearch61(16) with denovo models. Sequences were clustered against the 2013 Greengenes (13_8 release) ribosomal database's $97 \%$ reference data set. Sequences that did not match any entries in this reference were subsequently clustered into de novo OTUs at $97 \%$ similarity with UCLUST. Taxonomy was assigned to all OTUs using the RDP classifier (17) within QIIME and the Greengenes reference data set. OTU tables were subsequently subjected to abundance-based filtering, removing low-abundance OTUs representing less than $0.005 \%$ of total reads in the data set. Then, the OTU table was rarefied to a sequencing depth of 25000 per sample for subsequent analyses of alpha diversity and beta diversity. Alpha diversity (observed OTUs and Shannon) and beta diversity (PCoA analysis) were analyzed using the QIIME standard pipeline.

\section{Statistical Analysis}

The data are presented as the mean \pm SD. Chi-squared-tests were used to assess gender differences, and the Kolmogorov-Smirnow test was used to evaluate alpha diversity. Analysis of similarities (ANOSIM) analysis on beta diversity matrices was performed in QIIME to test for significant differences between the microbial communities. The significance of the ANOSIM test was assessed with 9999 permutations. Linear discriminant analysis effect size (LEfSe) was introduced to identify bacterial biomarkers for two groups, which was performed on the Galaxy web-based interface (http://huttenhower.sph.harvard.edu/galaxy). We used standard parameters except the alpha value for the factorial Kruskal-Wallis test among classes, and the threshold used to consider a discriminative feature for the logarithmic LDA score was set at $>2$.

\section{Results}

\section{Study population}

50 HSP children (HSP-C) and their mothers (HSP-M), together with 61 healthy children $(\mathrm{H}-\mathrm{C})$ and their mothers $(\mathrm{H}-\mathrm{M})$ were enrolled in this study to explore the gut microbiome. The age and gender of all enrolled subjects are summarized in Table 1. In this article, we defined each groups as HSP-C, HSP-M, HC, H-M, HSP-M + C (for HSP mother-child pairs) and H-M + C (for healthy mother-child pairs). 
Table 1

Characteristics of Study Participants

\begin{tabular}{|lllll|}
\hline Clinical Values & HSP-C (50) & H-C (61) & HSP-M (50) & H-M (61) \\
\hline Age Range (Mean), yrs & $3-13(6)$ & $2-10(5)$ & $26-40(35)$ & 27-42 (31) \\
\hline $\begin{array}{llll}\text { Gender } \\
\text { Female }\end{array}$ & 35 & 33 & 50 & 61 \\
\hline Male & 35 & $\mathrm{~N} / \mathrm{A}$ & $\mathrm{N} / \mathrm{A}$ \\
\hline $\begin{array}{l}\text { Abbreviations: HSP-C, children with Henoch-Schönlein Purpura; H-C, healthy children; HSP-M, mothers } \\
\text { of children with Henoch-Schönlein Purpura; H-M, mothers of healthy children. }\end{array}$ & \\
\hline
\end{tabular}

To discover the characteristic gut microbiome of HSP children, we analyzed the alpha diversity between HSP-C and $\mathrm{H}-\mathrm{C}$, which revealed a significant decrease in microbial diversity (Shannon index, $\mathrm{p}<0.05$, Fig. 1A) and richness (Ace index, $p<0.05$, Fig. 1D) in HSP-C. The beta diversity based on the unweighted UniFrac distance showed distinct composition of gut microbiome between HSP-C and $\mathrm{H}-\mathrm{C}(\mathrm{p}=0.01, \mathrm{r}=$ 0.357, ANOSIM, Fig. 2A). To further explore the gut microbial community features of HSP-C, the relative taxon abundance was assessed between HSP-C and H-C. At the phylum level, three predominant phyla, including Bacteroidetes, Firmicutes and Proteobacteria, were displayed in the gut microbiome of HSP-C. Although there is no difference in the classification of taxon, the relative abundance of Prevotella was significantly increased in HSP-C compare with H-C (Fig. 3A-B). The Venn diagram showed that 93 of 107 core taxa were common to both HSP-C and H-C. HSP-C possessed 2 unique taxa, inexistent in $\mathrm{H}-\mathrm{C}$ (Fig. 2D). These significant differences were further confirmed by LEfSe analysis, which identified 46 discriminative microbial signatures between HSP-C and H-C. The genera of Enterococcus, Megamonas, Fusobacterium, Veillonella and Streptococcus were significantly increased in HSP-C, and the genera of Dialister, Sutterella, Lachnospira, Haemophilus, Roseburia, Ruminococcus, Coprococcus, Oscillospira, Anaerostipes, Bilophila and Akkermansia were significantly decreased (Fig. 4A, SFigure 1).

\section{Mothers of HSP children harbor different gut microbiome compared with mothers of healthy control children}

We compared the alpha and beta diversity between HSP-M and H-M, which showed surprisingly distinct gut microbiome composition. Analysis of alpha diversity revealed a significant decrease in microbial diversity ( $p=0.3$, Shannon, Fig. 1B) and richness ( $p<0.05$, ACE, Fig. 1E) in HSP-M. The analysis of beta diversity based on the unweighted UniFrac distances showed that the gut microbiome of HSP-M was significantly different from that of H-M (ANOSIM, $r=0.143, p=0.01$, unweighted UniFrac, Fig. 2B). These significant differences were further confirmed by LEfSe analysis, which identified 24 discriminative microbial signatures (LDA score > 2) that varied significantly between HSP-M and H-M. At the genus level, 
a significant increase of the relative abundance of Prevotella and Megamonas and a significant reduction of the taxa of Bacteroides, Lachnospira, Streptococcus, Haemophilus, Akkermansia, Oscillospira, Kingella and Paludibacter were observed in HSP-M compared with H-M (LDA $>3$, Fig. 3A). All potential biomarkers $(\mathrm{LDA}>2)$ were shown in Fig. 4A and Figure S1.

\section{HSP children harbor unique microbial biomarkers although their gut microbiome is closely associated with that of their mothers}

In order to reveal the correlation of gut microbiota composition between HSP children and their mothers, we compared the gut microbiome among different groups. Comparing the results of alpha and beta diversity in two groups, we found the richness $(p<0.05)$ and diversity $(p<0.05)$ of HSP-M $+C$ was lower than that of the $\mathrm{H}-\mathrm{M}+\mathrm{C}$ (Fig. 1C, 1F), and the composition of gut microbiome was different between HSP$\mathrm{M}+\mathrm{C}$ and $\mathrm{H}-\mathrm{M}+\mathrm{C}$ (ANOSIM, $\mathrm{r}=0.218, \mathrm{p}=0.01$, unweighted UniFrac, Fig. $2 \mathrm{C}$ ). Subsequently, we analyzed the Venn diagrams which by $70 \%$ cutoff showed the similarity and difference of four groups. The taxon of Acidaminococcus and Roseburia were only found in HSP-M + C (Fig. 2D).

\section{The potential value of the discovered microbial biomarkers for risk assessment}

To evaluate the potential value of the identified microbial biomarkers for two levels of clinical discrimination: HSP-C vs. H-C and HSP-M vs. H-M, we constructed the receiver operating characteristic (ROC) curve and computed the area under the curve (AUC) value. The sensitivity and specificity for the biomarker models were estimated by identifying the cut-off point of the predicted probability that yielded the highest sum of sensitivity and specificity. For HSP-C vs. H-C, the ROC-plot AUC value was 0.909 (95\% Cl 78.94-99.31\%) with $96.92 \%$ sensitivity and 98\% specificity (Fig. 4B); For HSP-M vs. H-M, the ROC-plot AUC value was 0.450 (95\% $\mathrm{Cl} 34.71-84.43 \%$ ) with $53.97 \%$ sensitivity and $56 \%$ specificity (Fig. 4 C).

\section{Discussion}

Recent studies have established that the etiology of HSP includes both genetic and environmental components (18) (19). However, it is only the beginning to elucidate the environmental factors that may be involved in the development of HSP and the molecular mechanisms through which these factors function. The high incidence of HSP and the severe symptoms of purpura make it important to understand the microbiome of people with high incidence of purpura. In our previous study, we discovered the potential correlation of gut microbiota dysbiosis with HSP in children (8). Furthermore, multiple studies have indicated that in addition to genetic influences, the maternal gut microbiota may play an essential role in the occurrence of diseases in offspring during gestation and exhibits continuous correlations and long-term pathological consequences during development (9). However, it remains unclear whether the maternal gut bacterial communities have associations with that of the HSP children, 
and whether the HSP children harbor unique gut microbial taxon that may be the key players in pathogenesis. To the best of our knowledge, no previous studies have investigated the gut microbiome of HSP children and their mothers in parallel and evaluated their correlations.

Our study is pilot to examine the correlations between the microbiome profiles of HSP children and their mothers. We found that the gut microbiota of HSP children and their mother displayed a remarkable dysbiosis, including decreased bacterial diversity and richness (Fig. 1), as well as a different microbiota structure when compared with that of healthy children and their mother. Additionally, while there were close correlations between the microbiome profiles of mother-child pairs, the HSP children exhibited unique bacterial biomarkers. Finally, the identified bacterial biomarkers exhibited remarkable discriminatory power for differentiating HSP children from control children and their mothers. These biomarkers may serve as critical pathogenetic bacteria that deserve further investigations.

Consistent with our previous clinical studies on the gut microbiome of HSP children, a comparison of the gut bacterial community structures between HSP children and healthy controls in our study revealed significantly shifted microbiome profiles in the feces of HSP children and identified a set of bacterial biomarkers that varied significantly between the two groups, such as Enterococcus, which has been reported to be associated with immune diseases such as eczema (20). Similarly, Dialister and Roseburia were markedly decreased in the HSP-C. Roseburia also had been found decrease in patients with ulcerative colitis (21), which plays a major role in maintenance of colonic mucosal health and antiinflammation by producing butyrat $(22)(23,24)$.

Surprisingly, HSP-M showed significantly different gut microbiome with H-M, although both of them are basically "healthy" females. Interestingly, whether it is an HSP child or a healthy child, his/her gut microbiome composition is more similar to that of his/her mother. Each mother-child pair group seems to possess distinct core microbiome. These results indicate that gut microbiome can be vertically transmitted from the mother to the gut of offspring, which could partially explain the similarities in the gut microbiome profiles of the mother-child pairs (25). Further studies are required to identify the specific causes of these variations and associations.

We found HSP-C and HSP-M share common biomarkers, such as Prevotella and Megamonas, whereas Lachnospira, Haemophilus, and Akkermansia were decreased in both HSP-C and HSP-M group. Haemophilus also has been found decreased in the gut of rheumatoid arthritis patients (26) and food allergy of children (27). Although HSP-C and HSP-M share common biomarkers, the distinct gut bacterial biomarkers of HSP children also included four species showing increased abundance compare with their mother, namely, Enterococcus, Fusobacterium, Veillonella and Streptococcus. Notably, Streptococcus were found to be increased in HSP children but decreased in HSP-M. Enterococcus is a major commensal bacteria in the human digestive tract and also the main opportunistic pathogen in the human gut reports, which was reported to be related to acquired immunodeficiency syndrome (28), Henoch-Schönlein purpura (8), and Crohn's disease (29). A recent study suggested that Veillonella parvula strongly induces IL-6, a proinflammatory cytokine involved in the development of immune system diseases (29). 
Veillonella also has been associated with the development of a healthy immune system in infants (30). And gut Streptococcus has been reported can affect persistence and severity of atopic dermatitis during infancy (31).

A dysbiotic gut microbiota has been associated with allergic diseases (32). Based on the discovered biomarkers, HSP children and the mothers of HSP children could be separated from healthy children and their mothers with high sensitivity and specificity. The discriminatory power of these candidate biomarkers paves the way for establishing fecal microbiome tests for clinical diagnostic and prognostic screening for HSP. However, our study had some limitations. First, the cross-sectional nature of the study did not enable us to elucidate the mechanisms and longitudinal aspects of the correlations. Additional large cohort studies are needed to establish the time sequence and evaluate changes in the gut microbiome of mothers and children. Furthermore, the small sample size did not allow subgroup analysis to assess whether the correlations were consistent across different patients with HSP, as defined by factors such as severity and comorbidity. However, this does not detract from the potential value of these biomarkers for clinical validation and applications, which is currently being evaluated in a nested, casecontrolled study using a population-based cohort.

\section{Conclusions}

Significant differences in the gut bacterial composition were observed between HSP children and healthy children. The gut microbiome of HSP children was closely associated with that of their mothers, although children with HSP still had unique bacterial biomarkers. Dysbiosis of the maternal gut microbiota may play a critical role in increasing the risk of HSP in children. The identified similarities and differences in mother-child gut microbiome profiles are important for early assessment of risks and for planning personalized treatment and prevention strategies for HSP via microbiota modulation.

\section{Declarations}

\section{Ethics approval and consent to participate}

This study, which was approved by the Institutional Review Boards of Qilu Children's Hospital of Shandong University and Shandong Provincial Hospital Affiliated to Shandong University, began sample collection in July 2016. Written informed consents and questionnaire data sheets were obtained from all subjects (mothers) who visited the Institute of Child Health Care and agreed to serve as stool donors, in compliance with national legislation and the Code of Ethical Principles for Medical Research Involving Human Subjects of the World Medical Association (Declaration of Helsinki).

\section{Consent for publication}

Not applicable 


\section{Availability of data and materials}

All sequencing data associated with this study were uploaded to the NCBI SRA database (accession number: SUB 4652081). The webpage of the SRA database is https://www.ncbi.nlm.nih.gov/sra/.

\section{Competing interests}

All authors declare that there is no conflict of interests regarding the publication of this paper.

\section{Funding}

All phases of this study were supported by the Shandong Provincial Key Research and Development Program (Grant No. 2018CXGC1219) to Zhongtao Gai; the Weihai Technique Extension Project under contract No. 2016GNS023 (Lei Zhang); the Shandong Provincial Key Research and Development Program under contract No. 2016YYSP009 (Lei Zhang); Lei Zhang is also supported by the TaiShan Scholars Program of Shandong Province (No. tshw20120206) and TaiShan Industrial Experts Program (No. tscy20190612).

\section{Authors' contributions}

Prof Lei Zhang conceptualized and designed the study, drafted the initial manuscript, and reviewed and revised the manuscript. Profs Zhongtao Gai and Junjie Yang conceptualized and designed the study, coordinated and supervised data collection, and critically reviewed the manuscript for important intellectual content. Yi Liu, Ying Wang, Xingcui Wang, Xuemei Liu, Hongxia Zhang, Nan Shen designed the clinical settings, went through ethic evaluation process, performed consents and questionnaire data sheets with patients, collected clinical samples and patients' information, and reviewed and revised the manuscript. Changying Zhao, Jiaming Zhang, Bin Chen, designed the data collection instruments, collected data, carried out the initial analyses, and reviewed and revised the manuscript. All authors approved the final manuscript as submitted and agree to be accountable for all aspects of the work.

\section{Acknowledgements}

Not applicable

\section{References}

1. Reid-Adam J. Henoch-Schonlein purpura. Pediatrics in review. 2014;35(10):447-9. discussion 9.

2. Calvo-Rio V, Loricera J, Mata C, Martin L, Ortiz-Sanjuan F, Alvarez L, et al. Henoch-Schonlein purpura in northern Spain: clinical spectrum of the disease in 417 patients from a single center. Med (Baltim). 
2014;93(2):106-13.

3. Ozen S, Pistorio A, lusan SM, Bakkaloglu A, Herlin T, Brik R, et al. EULAR/PRINTO/PRES criteria for Henoch-Schönlein purpura, childhood polyarteritis nodosa, childhood Wegener granulomatosis and childhood Takayasu arteritis: Ankara 2008. Part II: Final classification criteria. Ann Rheum Dis. 2010;69(5):798-806.

4. Gulati S. Henoch-Schönlein Purpura: Springer Singapore; 2017.

5. Round JL, Palm NW. Causal effects of the microbiota on immune-mediated diseases. Science immunology. 2018;3(20).

6. Kinumaki A, Sekizuka T, Hamada H, Kato K, Yamashita A, Kuroda M. Characterization of the gut microbiota of Kawasaki disease patients by metagenomic analysis. Frontiers in microbiology. 2015;6:824.

7. Abrahamsson TR, Jakobsson HE, Andersson AF, Bjorksten B, Engstrand L, Jenmalm MC. Low diversity of the gut microbiota in infants with atopic eczema. J Allergy Clin Immunol. 2012;129(2):434-40. 40 e1-2.

8. Wang X, Zhang L, Wang Y, Liu X, Zhang H, Liu Y, et al. Gut microbiota dysbiosis is associated with Henoch-Schonlein Purpura in children. Int Immunopharmacol. 2018;58:1-8.

9. Ferretti P, Pasolli E, Tett A, Asnicar F, Gorfer V, Fedi S, et al. Mother-to-Infant Microbial Transmission from Different Body Sites Shapes the Developing Infant Gut Microbiome. Cell Host Microbe. 2018;24(1):133-45. e5.

10. Yassour M, Jason E, Hogstrom LJ, Arthur TD, Tripathi S, Siljander H, et al. Strain-Level Analysis of Mother-to-Child Bacterial Transmission during the First Few Months of Life. Cell Host Microbe. 2018;24(1):146-54. e4.

11. York A. Delivery of the gut microbiome. Nature reviews Microbiology. 2018;16(9):520-1.

12. Subspecialty Group of I, Society of P. Chinese Medical A. Editorial Board of Chinese Journal of P. [Evidence-based recommendations for the diagnosis and management in the children with HenochSchonlein purpura]. Zhonghua er ke za zhi = Chinese journal of pediatrics. 2013;51(7):502-7.

13. Findley K, Oh J, Yang J, Conlan S, Deming C, Meyer JA, et al. Topographic diversity of fungal and bacterial communities in human skin. Nature. 2013;498(7454):367-70.

14. Magoc T, Salzberg SL. FLASH: fast length adjustment of short reads to improve genome assemblies. Bioinformatics. 2011;27(21):2957-63.

15. Caporaso JG, Kuczynski J, Stombaugh J, Bittinger K, Bushman FD, Costello EK, et al. QIIME allows analysis of high-throughput community sequencing data. Nature methods. 2010;7(5):335-6.

16. Edgar RC. Search and clustering orders of magnitude faster than BLAST. Bioinformatics. 2010;26(19):2460-1.

17. Cole JR, Wang Q, Cardenas E, Fish J, Chai B, Farris RJ, et al. The Ribosomal Database Project: improved alignments and new tools for rRNA analysis. Nucleic acids research. 2009;37(Database issue):D141-5. 
18. Yang YH, Chuang YH, Wang LC, Huang HY, Gershwin ME, Chiang BL. The immunobiology of HenochSchonlein purpura. Autoimmun rev. 2008;7(3):179-84.

19. Chen JY, Mao JH. Henoch-Schonlein purpura nephritis in children: incidence, pathogenesis and management. World journal of pediatrics: WJP. 2015;11(1):29-34.

20. Hong PY, Lee BW, Aw M, Shek LP, Yap GC, Chua KY, et al. Comparative analysis of fecal microbiota in infants with and without eczema. PLoS One. 2010;5(4):e9964.

21. Machiels K, Joossens M, Sabino J, De Preter V, Arijs I, Eeckhaut V, et al. A decrease of the butyrateproducing species Roseburia hominis and Faecalibacterium prausnitzii defines dysbiosis in patients with ulcerative colitis. Gut. 2014;63(8):1275-83.

22. Tamanai-Shacoori Z, Smida I, Bousarghin L, Loreal O, Meuric V, Fong SB, et al. Roseburia spp.: a marker of health? Future microbiology. 2017;12:157-70.

23. O'Keefe SJ. Diet, microorganisms and their metabolites, and colon cancer. Nature reviews Gastroenterology hepatology. 2016;13(12):691-706.

24. Furusawa Y, Obata Y, Fukuda S, Endo TA, Nakato G, Takahashi D, et al. Commensal microbe-derived butyrate induces the differentiation of colonic regulatory T cells. Nature. 2013;504(7480):446-50.

25. Backhed F, Roswall J, Peng Y, Feng Q, Jia H, Kovatcheva-Datchary P, et al. Dynamics and Stabilization of the Human Gut Microbiome during the First Year of Life. Cell Host Microbe. 2015;17(6):852.

26. Zhang X, Zhang D, Jia H, Feng Q, Wang D, Liang $D$, et al. The oral and gut microbiomes are perturbed in rheumatoid arthritis and partly normalized after treatment. Nat Med. 2015;21(8):895-905.

27. Savage JH, Lee-Sarwar KA, Sordillo J, Bunyavanich S, Zhou Y, O'Connor G, et al. A prospective microbiome-wide association study of food sensitization and food allergy in early childhood. Allergy. 2018;73(1):145-52.

28. Zhou Y, Ou Z, Tang X, Zhou Y, Xu H, Wang X, et al. Alterations in the gut microbiota of patients with acquired immune deficiency syndrome. J Cell Mol Med. 2018;22(4):2263-71.

29. Mondot S, Lepage P, Seksik P, Allez M, Treton X, Bouhnik Y, et al. Structural robustness of the gut mucosal microbiota is associated with Crohn's disease remission after surgery. Gut. 2016;65(6):95462.

30. Poppleton DI, Duchateau M, Hourdel V, Matondo M, Flechsler J, Klingl A, et al. Outer Membrane Proteome of Veillonella parvula: A Diderm Firmicute of the Human Microbiome. Front Microbiol. 2017;8:1215.

31. Mee PY, Kang MJ, Lee SY, Yoon JS, Jung S, Cho HJ, et al. Gut Streptococcus species affect persistence and severity of atopic dermatitis during infancy. Journal of Allergy Clinical Immunology. 2018;141(2):AB232.

32. Tamburini S, Shen $\mathrm{N}, \mathrm{Wu} \mathrm{HC}$, Clemente JC. The microbiome in early life: implications for health outcomes. Nature medicine. 2016;22(7):713-22. 
Figures

A

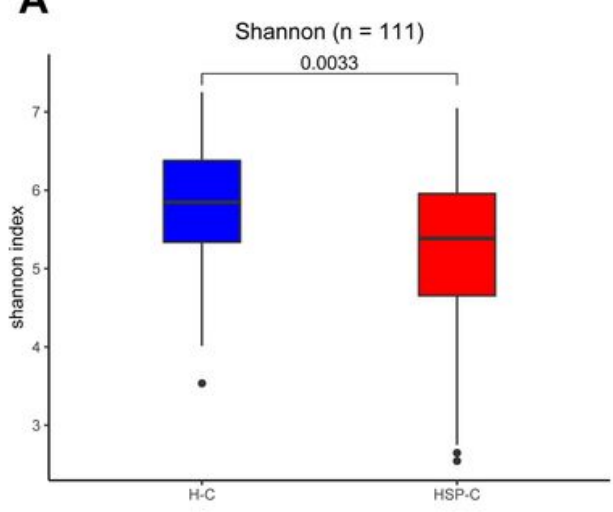

D

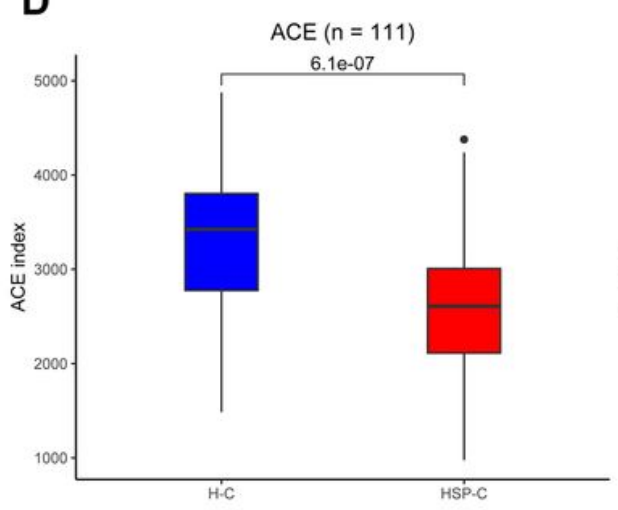

B

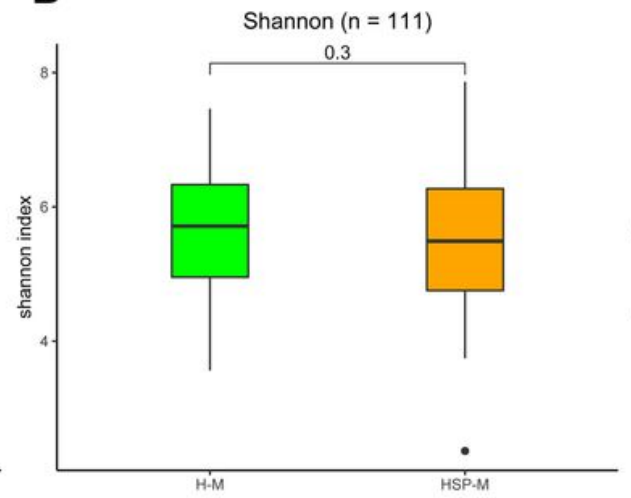

E

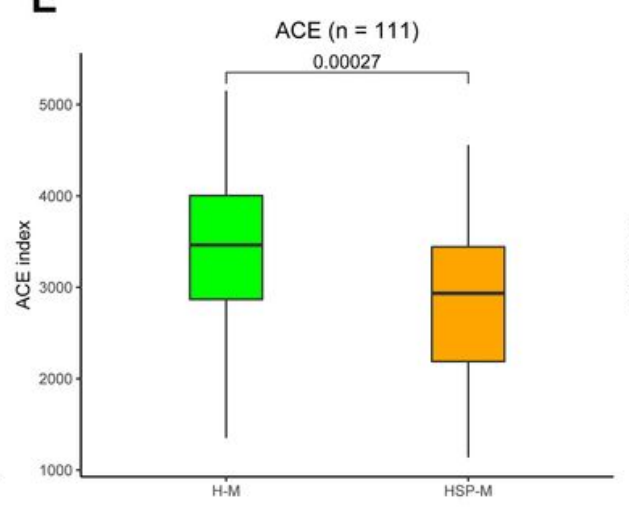

C

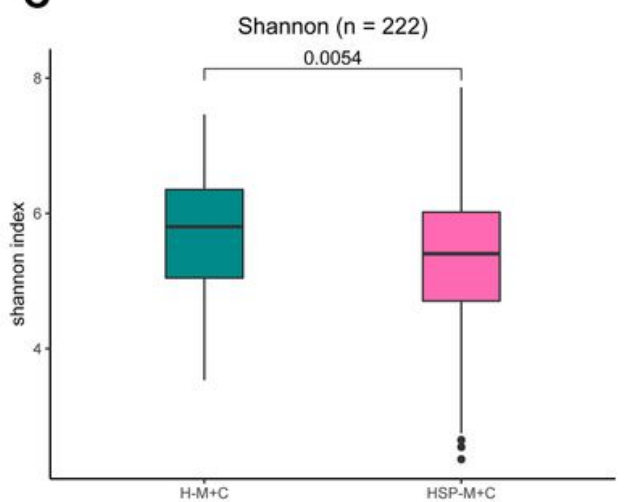

$\mathbf{F}$

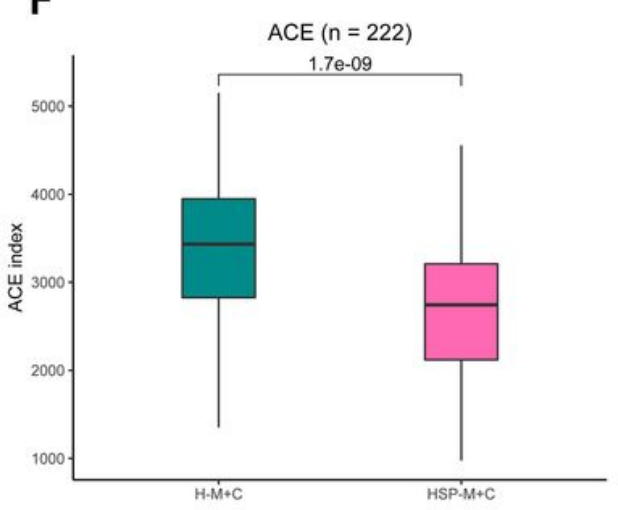

\section{Figure 1}

The diversity and richness of the gut microbiota in four groups. The Shannon index of the gut microbiome (A) in H-C and HSP-C, (B) in H-M and HSP-M, (C) in H-M+CandHSP-M+C. And the ACE index of the gut microbiome (D) in H-C and HSP-C, (E) in H-M andHSP-M, (F) in H-M+C and HSP-M+C. 
A

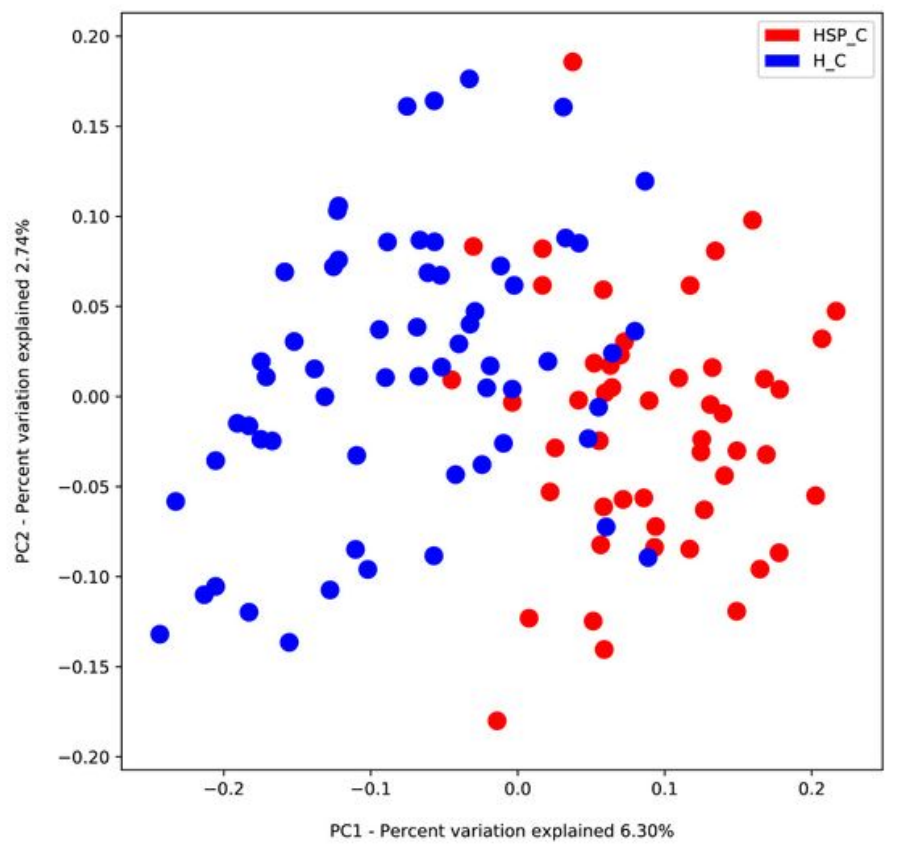

C

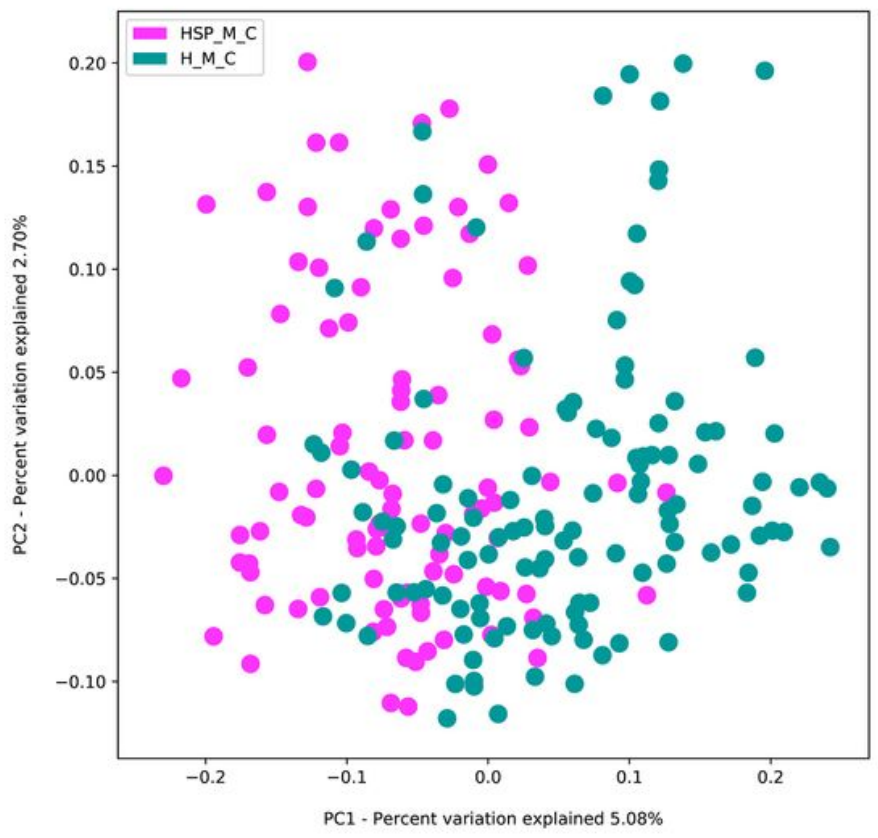

B

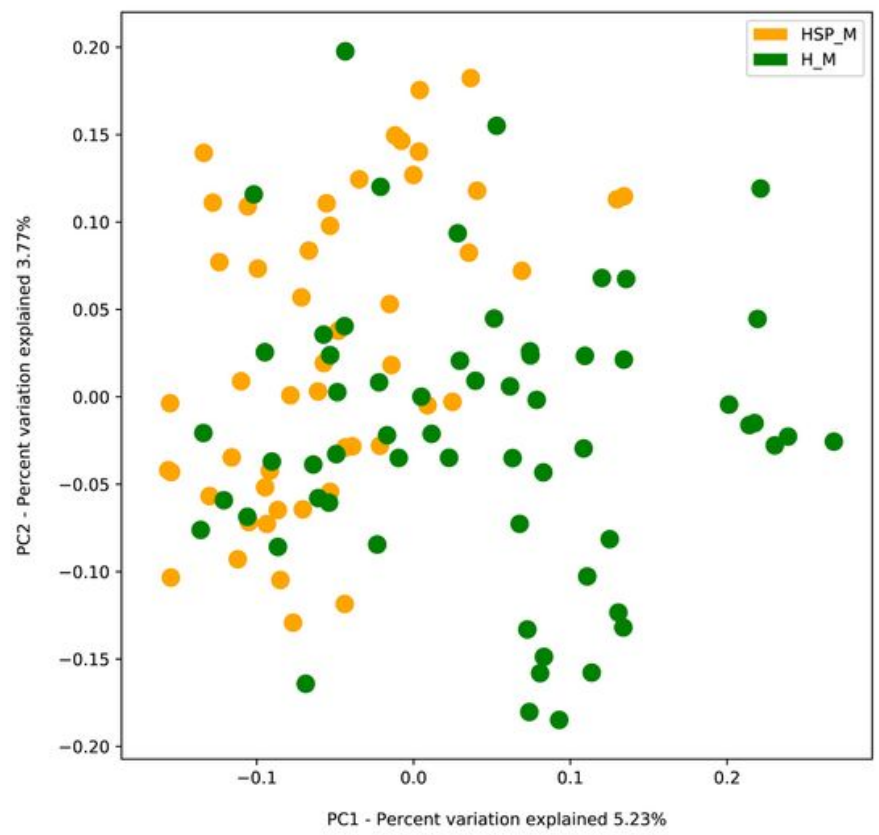

D

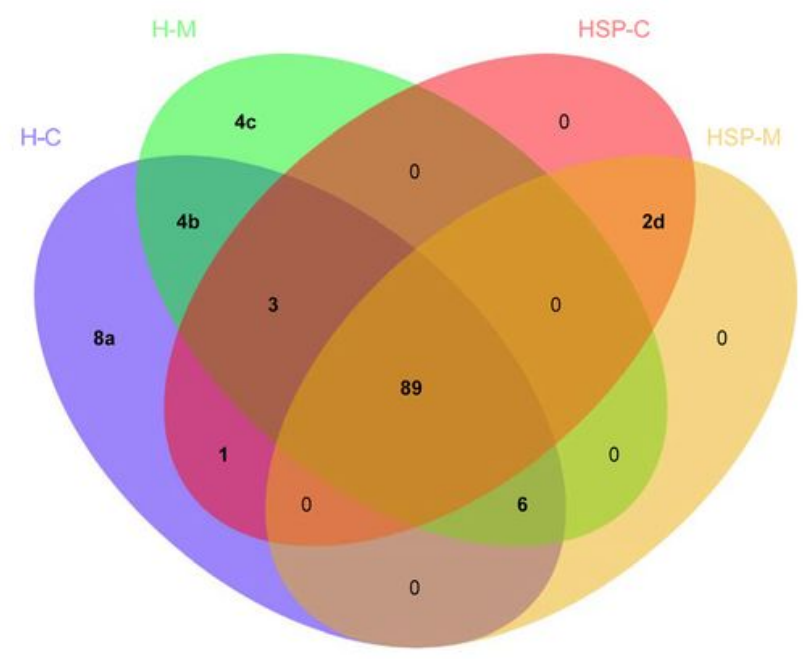

Figure 2

Microbiome community and Venn diagram analysis. PCoA of bacterial beta diversity based on the unweighted UniFrac distances. (a) HSP-C vs. H-C; (b) HSP-M vs. H-M (c) HSP-M+C vs. H-M+C. (d) Venn diagram displaying the degree of overlap of bacterial OTUs among HSP-C, HSP-M, H-C and H-M.Venn diagrams were constructed to evaluate the number and identity of the shared OTUs among groups, demonstrating that the similarity of the gut microbiome in the ASD-M+C group was higher than that in the $\mathrm{H}-\mathrm{M}+\mathrm{C}$ group 
A

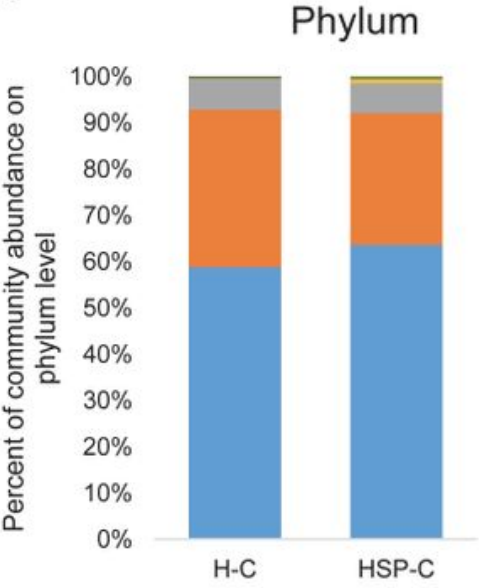

C

Phylum

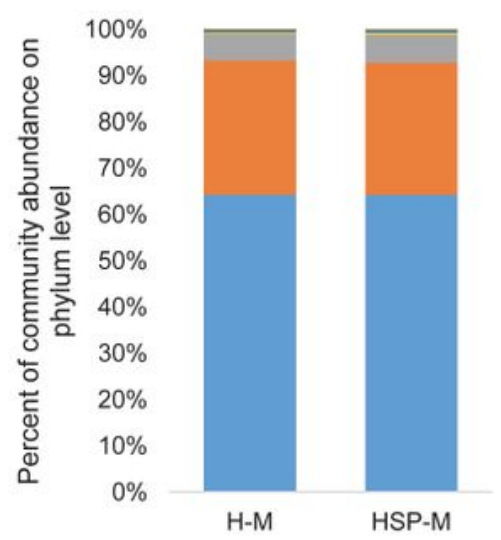

- Others

= Spirochaetes

- Verrucomicrobia

- Actinobacteria

" Tenericutes

- Cyanobacteria

w Fusobacteria

= Proteobacteria

w Firmicutes

- Bacteroidetes

E

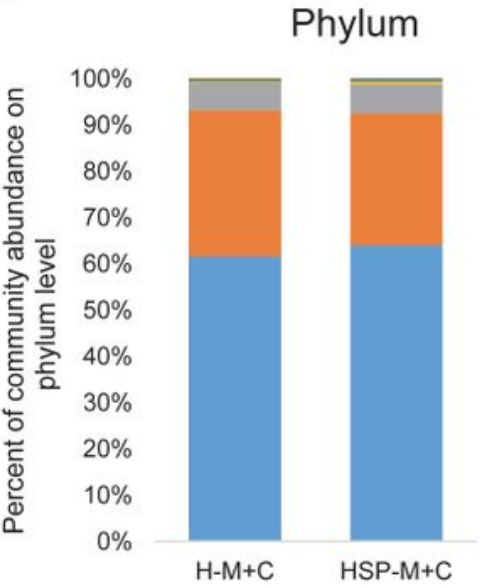

= Others

n Synergistetes

- Verrucomicrobia

- Tenericutes

- Actinobacteria

- Cyanobacteria

in Fusobacteria

w Proteobacteria

w Firmicutes

m Bacteroidetes
B
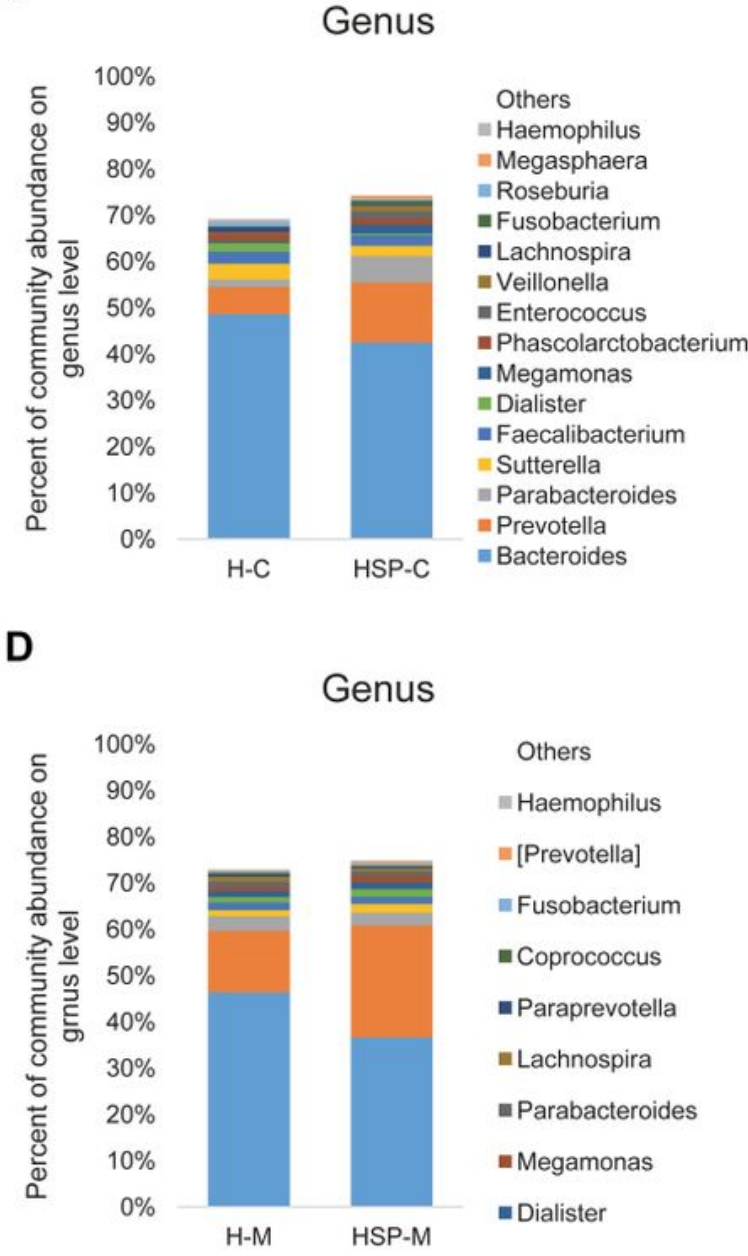

$\mathbf{F}$

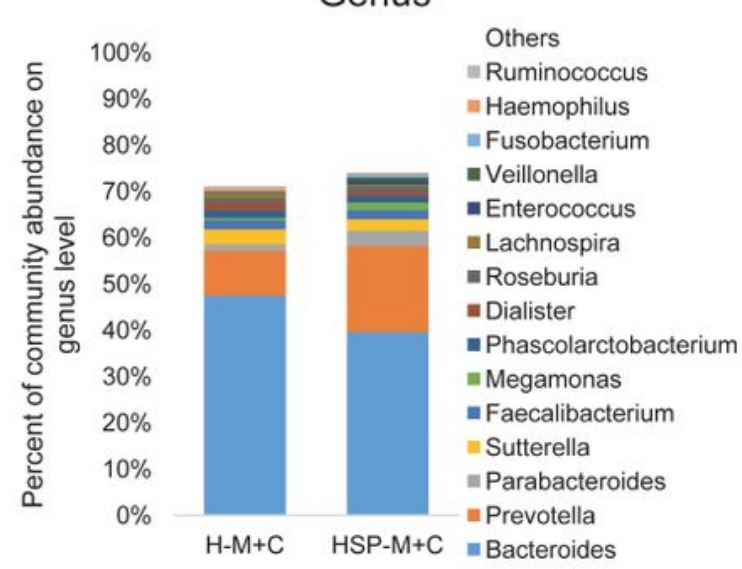

\section{Figure 3}

Comparison of relative taxa abundance among four groups at phylum and genuslevel. Comparison of relative taxa abundance between different groups at the phylum $(A, C, E)$ and genus $(B, D, F)$ level (average of eachgroup). (A, B) H-C vs. HSP-C; (C, D) H-M vs. HSP-M; (E, F) H-M+C vs. HSP-M+C. 


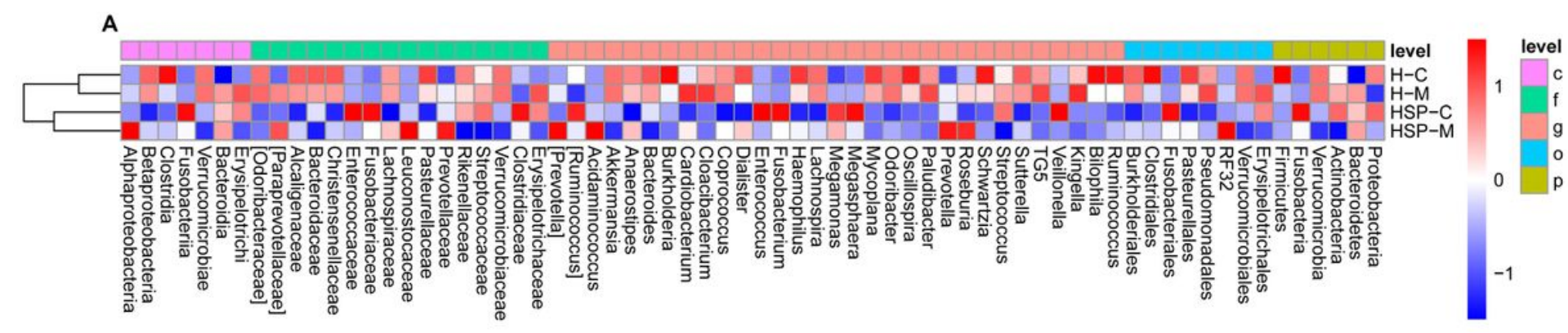

B

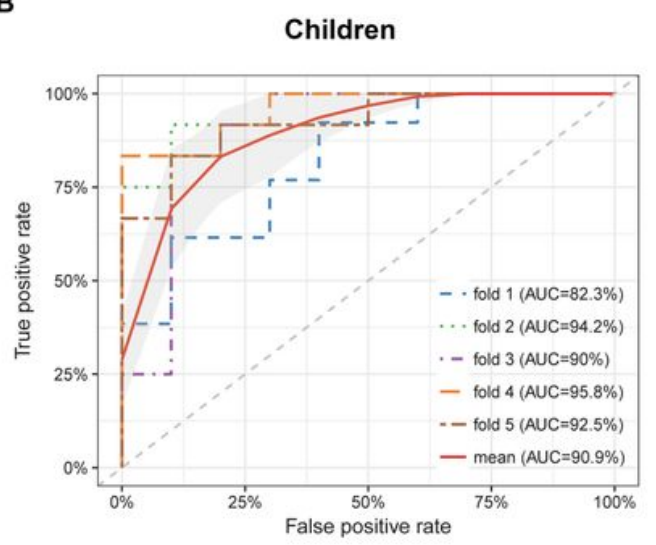

C

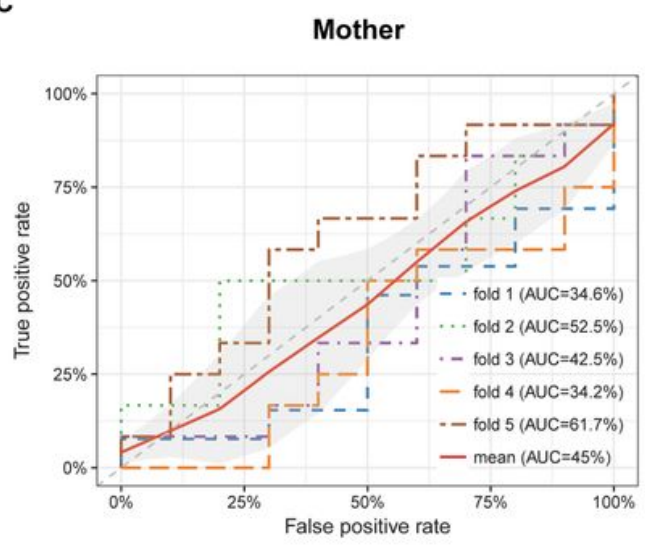

Figure 4

The Relative Abundance of the OTUs and Receiver Operating Characteristic Curves. The relative abundance of the top 68 most different OTUs across groups at the criteria of $p$ value $<0.1$ by Wilcoxon rank sum test. The abundance profiles are transformed into $Z$ scores by subtracting the average abundance and dividing the standard deviation of all samples. $Z$ score is negative (shown in blue) when the row abundance is lower than the mean. OTUs at $p$ value $<0.01$ are marked with red symbol, $p$ value $<$ 0.05 with green symbol. Receiver operating characteristic curves (b) for HSP-C vs. H-C; (c) for HSP-M vs.H-M were determined using the OTUs biomarkers and sequencing datasets and a supervised learning approach with random forest algorithm.

\section{Supplementary Files}

This is a list of supplementary files associated with this preprint. Click to download.

- SFigure1.tif 